\title{
Real Values of the $W$-Function
}

\author{
D. A. BARRY and P. J. CULLIGAN-HENSLEY \\ University of Western Australia \\ and
}

S. J. BARRY

Griffith University

\begin{abstract}
Approximations for real values of $W(x)$, where $W$ is defined by solutions of $W \exp (W)=x$, are presented. All of the approximations have maximum absolute $(|W|>1)$ or relative $(|W|<1)$ errors of $O\left(10^{-4}\right)$. With these approximations an efficient algorithm, consisting of a single iteration of a rapidly converging iteration scheme, gives estimates of $W(x)$ accurate to at least 16 significant digits (15 digits if double precision is used). The Fortran code resulting from the algorithm is written to account for the different floating-point-number mantissa lengths on different computers, so that $W(x)$ is computed to the floating-point precision available on the host machine.
\end{abstract}

Categories and Subject Descriptors: G.1.2 [Numerical Analysis]: Approximation-nonlinear approximation; G.1.5 [Numerical Analysis]: Roots of Nonlinear Equations - iterative methods

General Terms: Algorithms

Additional Key Words and Phrases: W-function

\section{INTRODUCTION}

The function defined by (1) below was first discussed by Euler [1921], and later by Pólya and Szegö [1972, part (3), prob. 209]. de Bruijn [1958] explored the asymptotic behavior of the W-function. Green and Knuth [1981] used de Bruijn's analysis as an archetypal example of "bootstrapping." Similarly, Oliver [1990] used a variant of (1) to demonstrate the derivation of asymptotic solutions of transcendental equations. In a practical application, the $W$-function was found recently to be an approximation describing water infiltration into a dry soil [Barry et al. 1995].

Authors' addresses: D. A. Barry, Department of Environmental Engineering, Centre for Water Research, The University of Western Australia, Nedlands, Western Australia 6907; email: barry@cwr.uwa.edu.au; P. J. Culligan-Hensley, Department of Civil and Environmental Engineering, Massachusetts Institute of Technology, Cambridge, MA 02139-4307; S. J. Barry, Faculty of Environmental Sciences, Griffith University, Nathan, Queensland 4111 Australia.

Permission to make digital/hard copy of part or all of this work for personal or classroom use is granted without fee provided that copies are not made or distributed for profit or commercial advantage, the copyright notice, the title of the publication, and its date appear, and notice is given that copying is by permission of ACM, Inc. To copy otherwise, to republish, to post on servers, or to redistribute to lists, requires prior specific permission and/or a fee.

(C) 1995 ACM 0098-3500/95/0600-0161 $\$ 03.50$ 


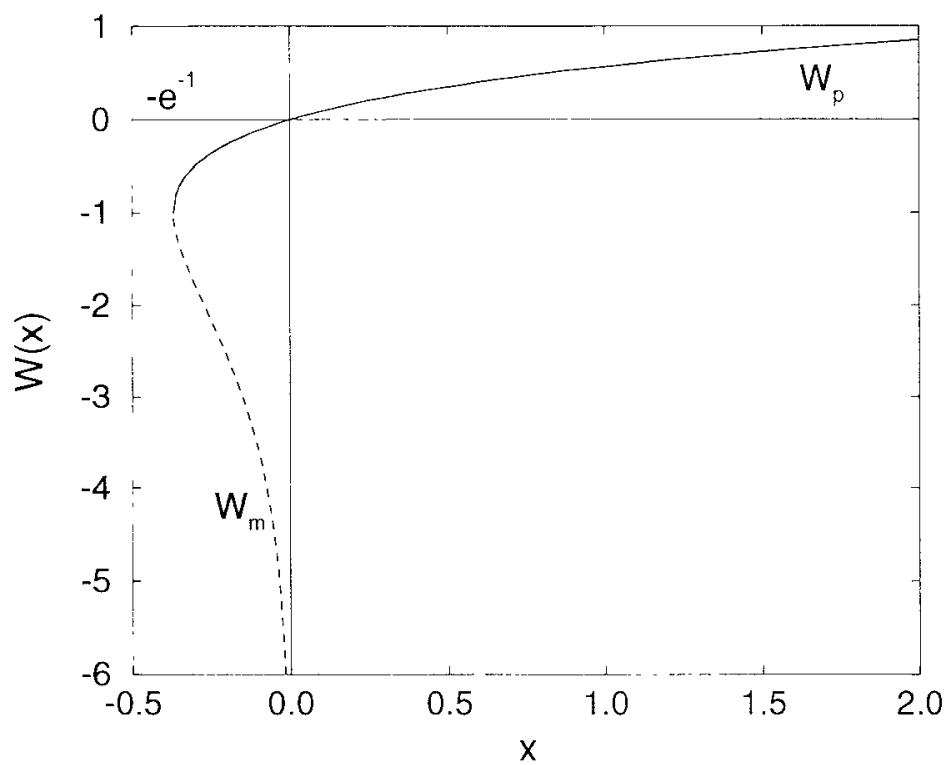

Fig. 1. W-function, showing the two branches, $W_{p}(W \geq-1)$ and $W_{m}(W<-1)$, considered in the text.

The approximations presented below can be used to solve equations of the form

$$
W B^{W}=x, \quad B>0, \quad x>\frac{-\exp (-1)}{\ln (B)}
$$

and

$$
W^{W}=x, \quad \ln (x) \geq-\exp (-1),
$$

since simple variable changes reduce these equations to the form of (1).

Fritsch et al. [1973] provided an algorithm to compute $W(x)$ that, when used in conjunction with an iteration scheme, was shown to give estimates in the range $0.01 \leq x \leq 1000$ with relative error of $O\left(10^{-6}\right)$. However, real values of $W(x)$ exist for $x \leq 0$ as well as for $x \geq 0$. Also, their iteration scheme is not suitable to compute $W(x)$ for $x \rightarrow 0$ or $-\exp (-1)$, as discussed below. For $x \geq 0$ and $x=-\exp (-1)$, the function is single valued, while for $-\exp (-1)<x<0$, it is double valued, as shown in Figure 1. The $W$-function behavior shown in Figure 1 is most easily determined by consideration of $x(W)$ rather than $W(x)$. For $W>0$, the product $W \exp (W)$ produces a single $x$. However, $W \exp (W)$ is zero for $W=0$ or $W \rightarrow-\infty$, showing that $W(x)$ is double valued for $W<0$. The minimum possible $x(W)$ is calculated as

$$
\frac{d x}{d W}=\exp (W)(1+W) \text {. }
$$

This expression is zero for $W \rightarrow-\infty\left(x \rightarrow 0^{-}\right)$or $W=-1(x=-\exp (-1))$. No real values of $W$ exist for $x<-\exp (-1)$. 
Our aims are (1) to produce closed-form approximations for $W(x)$ and (2) to provide an algorithm for calculating $W$ automatically to, or very near to, the floating-point precision of the host computer. The basic approach followed is to find approximations for $W(x)$, accurate to $O\left(10^{-4}\right)$. These are developed using various functional forms, including continued fractions and asymptotic expansions. Using these approximations, a single pass of the iterative improvement scheme of Fritsch et al. [1973] gives 16-significant-digit accuracy (typical Fortran douple precision). A second iteration gives 64-digit accuracy, and so on for further iterations.

\section{APPROXIMATIONS}

The function $W(x), x$ and $W$ both assumed real, is defined by

$$
W \exp (W)=x, \quad x \geq-\exp (-1)
$$

For $x \rightarrow 0^{-}$, the left-hand side of (1) can approach 0 in two ways, that is, if $W \rightarrow 0^{-}$or if $W \rightarrow-\infty$. This behavior is the cause of the dual values of $W$ for $-\exp (-1)<x<0$. For convenience, we denote the upper branch (i.e., $W \geq$ -1 in Figure 1) as $W_{p}$ and the lower branch as $W_{m}$. Each case is treated separately below. Before proceeding, however, note that writing (1) as $W=x$ $\exp (-W)$ yields, after repeated substitution,

$$
W=x \exp (-x \exp (-x \exp (-x \cdots,
$$

where the ellipsis indicates that the given form of the function continues indefinitely. Similarly, by taking logarithms of both sides of (1), there results

$$
W=\ln \frac{x}{\ln \frac{x}{\ln \frac{x}{\vdots}}} .
$$

Equation (2) was considered for use in approximating $W_{p}$ around $x=0$; however, continued fraction approximations are more effective computationally. Truncated forms of (3), on the other hand, were found to be useful for estimating $W_{p}$, for $x$ large, and $W_{m}$, for $x \rightarrow 0^{-}$, as shown below. An alternative approach is that of de Bruijn [1958], who gives an asymptotic expansion for $W(x)$ valid as $x \rightarrow \infty$. The relationship between the magnitude of $x$ and the accuracy of de Bruijn's expansion has not been explored. de Bruijn [1958] shows this series to be absolutely convergent for large $x$, but no general formula to calculate the series coefficients was presented, thus limiting the practical application of his result.

\subsection{Approximations for $W_{p}$}

An expansion of (1) around $x=-\exp (-1)$ yields (for both $W_{p}$ and $W_{m}$ )

$$
\begin{aligned}
W_{\left[\begin{array}{c}
p \\
m
\end{array}\right]=} & -1[ \pm] \sqrt{\eta}-\frac{\eta}{3}[ \pm] \frac{11}{72} \eta \sqrt{\eta} \\
& -\frac{43}{540} \eta^{2}[ \pm] \frac{769}{17,280} \eta^{2} \sqrt{\eta}-\frac{221}{8,505} \eta^{3}[ \pm] \cdots
\end{aligned}
$$


Table I. Constants Used in the Approximations Discussed in the Text

\begin{tabular}{ccc}
\hline Constant & Value & Equation \\
\hline$a$ & 4612634277343749 & $(6)$ \\
$b$ & 109556884765625 & $(6)$ \\
$a_{1}$ & 1.124491989777808 & $(8)$ \\
$b_{1}$ & 0.4225028202459761 & $(8)$ \\
$a_{2}$ & 127.0471381349219 & $(10)$ \\
$a_{3}$ & 0.5043921323068457 & $(12)$ \\
\hline
\end{tabular}

where $\eta=2+2 \exp (1) x$. Both of the series for $W_{p}$ and $W_{m}$ are identical except for sign changes. The first term in this expansion can be derived by noting that $W=-1$ at $x=-\exp (-1)$, and then by substituting $W=-1+$ $\delta_{W}$ and $x=-\exp (-1)\left(1-\delta_{x}\right)$ into (1), where $\delta_{W}$ and $\delta_{x}$ are perturbation parameters. A Taylor series expansion then shows that $\delta_{W}= \pm \sqrt{2 \delta_{x}}=$ $\pm \sqrt{\eta}$. This establishes that the series expansion of $W$ about $x=-\exp (-1)$ is in powers of $\sqrt{\eta}$. Higher-order terms can be found by assuming a series of this form, expanding as a Taylor series in $\delta_{x}$, and solving sequentially for the coefficient of each term. The series in (4) can be converted to a continued fraction [Lorentzen and Waadeland 1992], the latter form being more suitable for developing approximations to $W$. In truncated continued fraction form, (4) becomes

$$
W_{\left[\begin{array}{c}
p, 1 \\
m
\end{array}\right]}=-1+\frac{\sqrt{\eta}}{[ \pm] 1+\frac{\sqrt{\eta}}{3+D}},
$$

where

$$
D=\frac{n_{1} \sqrt{\eta}}{n_{2}+\sqrt{\eta}}
$$

with $n_{1}=135 / 83$ and $n_{2}=[ \pm] 360 / 83$. Equation (5) was derived from a series expansion about $x=-\exp (-1)$, so it will lose accuracy increasingly for increasing $x$. However, (5) can be used to approximate $W_{p}(x)$ over the range $-\exp (-1) \leq x \leq 20$ by adjusting $n_{1}$ and $n_{2}$. If we redefine $n_{1}$ to be

$$
n_{1}=\frac{4-3 \sqrt{2}+n_{2}(2 \sqrt{2}-3)}{\sqrt{2}-2}
$$

then (5) is exact for $W_{p}$ at both $x=-\exp (-1)$ and $x=0$. An accurate approximation for $W_{p}(x)$ in the range of interest was found by taking $n_{2}$ to be a function of the form

$$
n_{2}=a \sqrt{\sqrt{\eta+b}}
$$

where $a$ and $b$ are the constants listed in Table I. These constants were determined by minimizing the maximum relative error, that is, $\mid\left(W_{p, 1}-\right.$ $W) / W \mid$, over the range $-\exp (-1) \leq x \leq 20$. Because $W_{p}$ increases relatively 


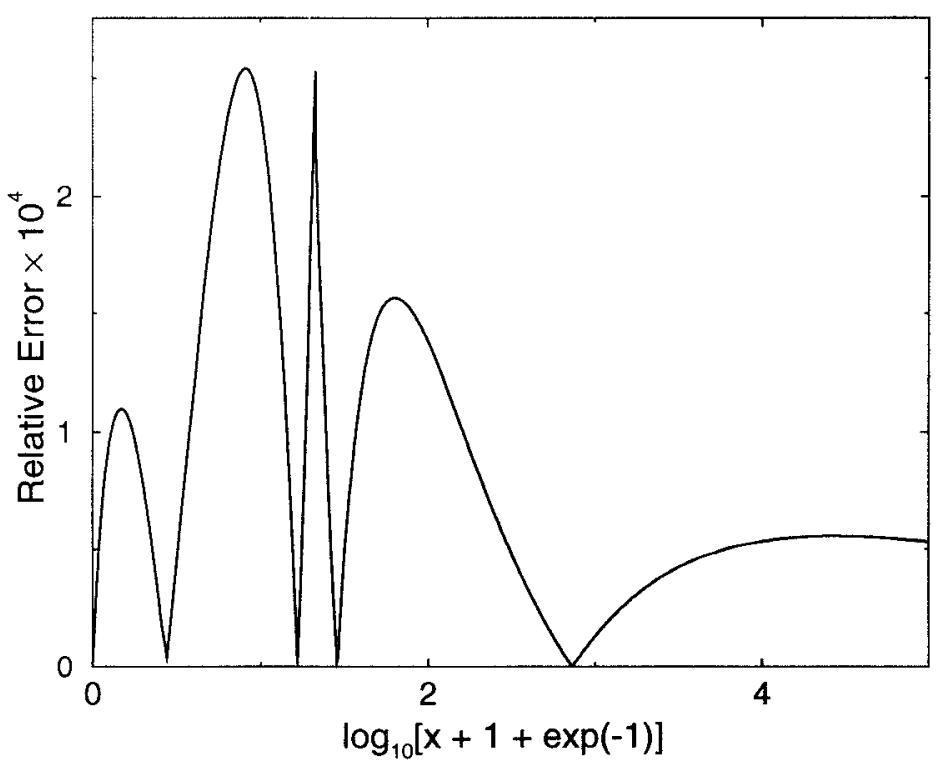

Fig. 2. Relative error of the approximations for $W_{p}$ given by (5) and (7).

slowly with $x$, both the maximum relative error and the maximum absolute error, $\left|W_{p, 1}-W\right|$, are $O\left(10^{-4}\right)$.

For $x>20$, we make use of the form (3). Any approximation based on (3) will yield the correct asymptotic behavior for $W$ as $x \rightarrow-\infty$. In particular,

$$
W_{p, 2}=\ln \frac{x}{\ln \frac{x}{(\ln x)^{h}}},
$$

where

$$
h=\exp \left[\frac{-a_{1}}{b_{1}+\ln (x)}\right]
$$

provides a satisfactory approximation to $W(x)$. The functional form for $h$ was determined by considering the difference, as a function of $x(>20)$, between (7) and exact solutions to (1). The constants $a_{1}$ and $b_{1}$, given in Table I, were fitted by minimizing the absolute error, $\left|W_{p, 2}-W\right|$, in the range $x>20$. This value of $x$ was chosen to match the absolute error due to (5); that is, (7) has a maximum absolute error of $O\left(10^{-4}\right)$. When computing $W_{p}$, we use (5) for $x \leq 20$, and (7) for $x>20$.

The accuracy of the approximations given by (5) and (8) is shown in Figure 2 , where the maximum relative error of the approximations is seen to be about $2.5 \times 10^{-4}$. With a single pass of the iteration scheme (13), both approximations are accurate to at least 16 significant digits at $x=20$, so the combination of both approximating functions is continuous to this number of digits. 


\subsection{Approximations for $W_{m}$}

In this case our procedure for developing an approximation for $W$ is slightly different. It is readily confirmed that

$$
W_{m, 1}=-1-t-\frac{2}{x_{1}}\left(1-\frac{1}{1+x_{1} \sqrt{\frac{t}{2}}}\right),
$$

where $t=-1-\ln (-x)$, reproduces the first term in both the $x \rightarrow-\exp (-1)$ and $x \rightarrow 0^{-}$expansions for $W_{m}$, regardless of the value of the constant $x_{1}$. That is, (9) is an interpolation between these limits. We therefore treat $x_{1}$ as a variable and calculate its series expansion about $x=-\exp (-1)$. This series expansion can then be expressed, approximately, in truncated continued fraction form as

$$
x_{1}=\frac{1}{3}-\frac{t}{270+a_{2} \sqrt{t}},
$$

where $a_{2}$ is a fitting constant to be determined. We choose $a_{2}$ (Table I) so that (9) produces $O\left(10^{-4}\right)$ absolute error estimates of $W_{m}$ in the range $-\exp (-1) \leq x \leq-\exp (-9)$.

For $-\exp (-9)<x<0$, we revert to a truncation of (3),

$$
W_{m, 2}=\ln \frac{x}{\ln \frac{-x}{g}},
$$

where

$$
g=\left(1+a_{3} t\right)\left(\sqrt{\eta}+\frac{\eta}{3}\right)+1
$$

The functional form in (12) was determined by considering the difference between (11) and $W$ calculated from (1). Again, $a_{3}$ in (12) is a fitting constant. By choosing $a_{3}$ as listed in Table $I$, we can approximate $W_{m}$ by (11) for $-\exp (-9)<x<0$, with maximum absolute error of $O\left(10^{-4}\right)$. The accuracy of the $W_{m}$ approximations is shown in Figure 3. Once again, both approximations are accurate to at least 16 significant digits at $x=-\exp (-9)$ with a single pass of (13) and so are continuous there to at least this number of digits.

\section{ITERATIVE SCHEME}

An efficient iteration scheme to compute more-accurate estimates of $W(x)$ based on a given initial guess has been described by Fritsch et al. [1973]. Given an approximation, $W_{n}$, to $W$, improved approximations are calculated using the iteration formula

$$
W_{n+1}=W_{n}\left(1+e_{n}\right),
$$




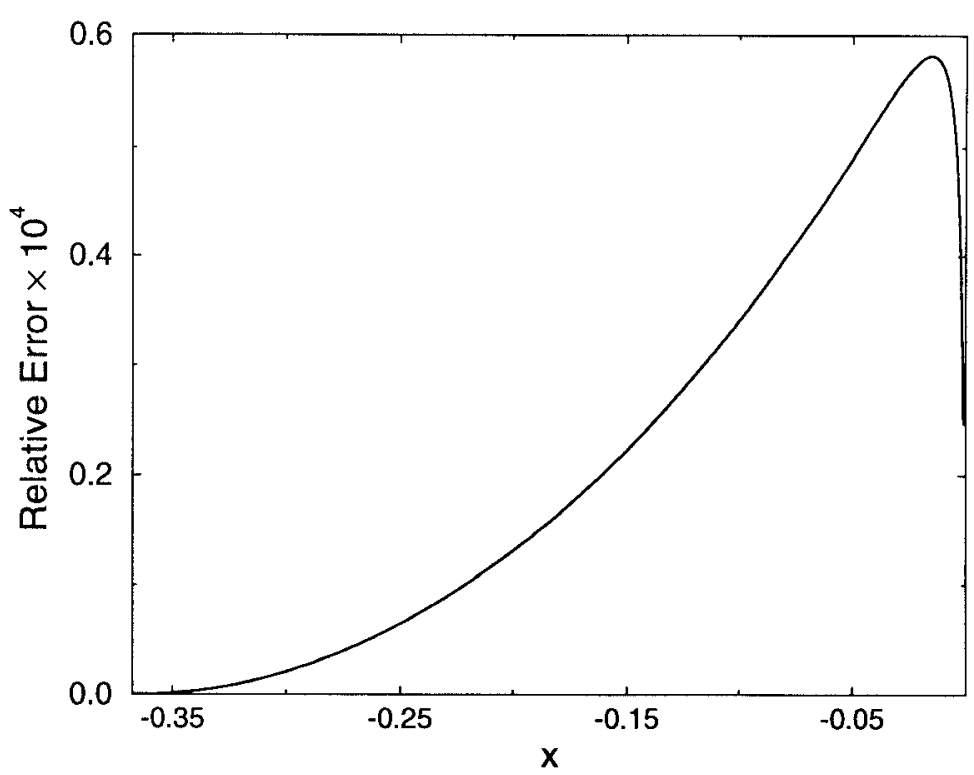

Fig. 3. Relative error of the approximations for $W_{m}$ given by (9) and (11).

where $n$ is the iteration count, $e_{n}$ is given by

$$
e_{n}=\left(\frac{z_{n}}{1+W_{n}}\right)\left[\frac{2\left(1+W_{n}\right)\left(1+W_{n}+\frac{2 z_{n}}{3}\right)-z_{n}}{2\left(1+W_{n}\right)\left(1+W_{n}+\frac{2 z_{n}}{3}\right)-2 z_{n}}\right],
$$

and

$$
z_{n}=\ln \frac{x}{W_{n}}-W_{n}
$$

The error term from this iteration is $O\left(e_{n}^{4}\right)$ [Fritsch et al. 1973]. With this iteration scheme, and the $O\left(10^{-4}\right)$ initial guesses given by the various approximations described above, a single iteration is sufficient to produce approximations to $W(x)$ correct to $O\left(10^{-16}\right.$ ) (relative or absolute error, as discussed above).

Most computers represent single-precision floating-point numbers using a 24-bit mantissa, or around 7 significant digits. Similarly, the mantissa for double-precision numbers is often 53 bits, the IEEE standard. In the latter representation, floating-point numbers are accurate to at most 16 significant digits. Thus, for most machines a single iteration using (13) gives results accurate to the number of significant digits available, remembering that at least 1 bit of precision is likely to be lost due to rounding error. For machines with a longer mantissa length, two iterations of (13) will produce results accurate to $O\left(10^{-64}\right)$. More-accurate estimates can be computed using further 
iterations, but it is unlikely that such accuracy is needed for most purposes. The Fortran code discussed below uses one or two iterations of (13) based on the bit-length of the mantissa of the machine being used.

Estimates based on (13) behave as outlined here except for $W_{p}$ in the region $x=0$, and both $W_{m}$ and $W_{p}$ near $x=-\exp (-1)$ where the scheme suffers because of rounding error. For this reason, $W_{p}$ is estimated using a continued fraction around $x=0$, that is,

$$
W_{p}=\frac{x\left(60+114 x+17 x^{2}\right)}{60+174 x+101 x^{2}}, \quad|x|<x_{0} .
$$

Equation (14) is exact at $x=0$ and becomes increasingly less accurate with increasing distance from the origin. The range over which (14) will apply, that is, the value of $x_{0}$, will vary for different word lengths used for storing floating-point numbers. The truncation error of (14) is $133 x^{6} / 720$. Thus, the error of (14) will increase with $x_{0}$ according to this size of the truncation term, that is, according to the magnitude of $133 x_{0}^{6} / 720$. Clearly, $x_{0}$ should be chosen such that $133 x_{0}^{6} / 720 \approx 2^{1-b i t s}$ (where bits is the number of bits in the floating-point-number mantissa of the host machine) if floating-point precision of the host machine is to be maintained (assuming 1 bit is lost to rounding). This condition is satisfied if $x_{0}$ is calculated using

$$
2 x_{0}^{6}=2^{1-b \imath t s} .
$$

Similarly, to overcome the rounding error due to (13) near $x=-\exp (-1)$ we replace the factor $D$ in (5) by

$$
D=\frac{\sqrt{\eta}}{[ \pm] \frac{8}{3}+\frac{\sqrt{\eta}}{\frac{135}{83}+\frac{\sqrt{\eta}}{[ \pm] \frac{166}{39}+\frac{3167}{3549} \sqrt{\eta}}}},
$$

so that the absolute value of truncation error of (5) is now approximately $4 \times 10^{-5} \eta^{7 / 2}$ for either $W_{p}$ or $W_{m}$. Then, proceeding as for $x_{0}$ above, with this $D$, (5) is used to calculate both $W_{p}$ and $W_{m}$ for $x$ in the range

$$
-\exp (-1)<x<-\exp (-1)\left[1-17 \times 2^{2(1-b i t s) / 7}\right]
$$

The coefficient of $x_{0}^{6}$ in (15) and that of $2^{2(1-b \imath t s) / 7}$ in (16) were fixed by numerical experimentation.

\section{TESTING}

The various approximations presented here were tested using the extendedprecision capability of the symbolic algebra program Maple V [Char et al. 1991]. Extended precision (50 digits) was used so that the true behavior of the approximations could be assessed without the interference of rounding errors. 

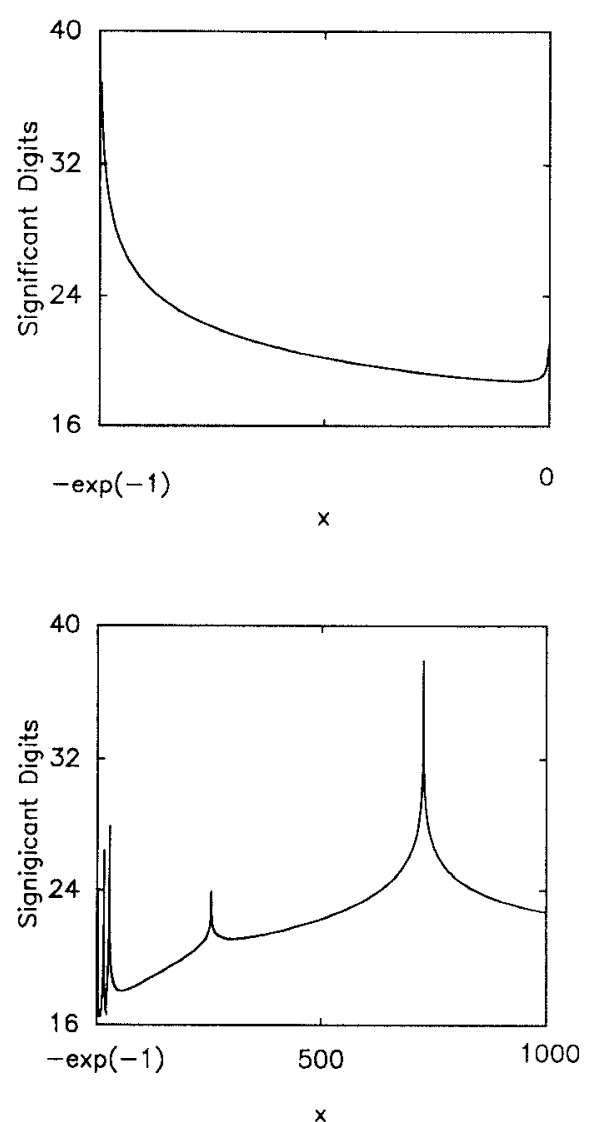

Fig. 4. Number of significant digits as determined by (17) for the approximations to $W_{m}$, with a single pass of the iterative improvement scheme (13).

Fig. 5. Number of significant digits as determined by (17) for the approximations to $W_{p}$ with a single pass of the iterative improvement scheme (13), over the range $-\exp (-1) \leq x \leq$ 1000 .

In Figures 4 and 5, we display the number of significant digits produced by the approximations for $W$ coupled with a single iteration of the scheme (13). In both figures the number of significant digits is found using

$$
\text { Significant digits }=-\log _{10}\left|\frac{W_{\text {approx }}-W}{W}\right|
$$

where $W_{\text {approx }}$ represents the various approximations given above. The exact values of $W$ in (17) were computed using Maple's built-in $W$-function (apparently based on the Fritsch et al. [1973] algorithm) for $W_{p}$ for $-\exp (-1)+$ $0.0017 \leq x \leq 2 \times 10^{5}$. Otherwise, (1), or an equivalent form of it, was solved using Maple's built-in root-finding routine. Likewise, $W_{m}$ values were determined by direct root-finding. Spikes in the figures occur around points where the approximation for $W$ is exact, in which case the right side of (17) becomes infinite. In Figure 4 it appears that there is a single spike in the neighborhood of $x=0$. This is an artifact of the discretization used in obtaining the data used in Figure 4. The scale used in the figure is such that the behavior for $x$ close to 0 , for example, $x>-\exp (-9)$, cannot be discerned. However, we have checked the algorithm's results in this region and have confirmed 
that the number of significant digits as $x \rightarrow 0^{-}$never decreases below that shown already in the figure. In Figure 5 we display only the range - $\exp (-1)$ $\leq x \leq 1000$. As was the case for Figure 4, we checked the region outside the range shown (i.e., $x>1000$; cf. Figure 2) and confirmed that the number of significant digits given by the approximation is always better than 16 . Due to rounding error, the Fortran-coded version of the algorithm (see below) produces results that are accurate to at least 15 digits. This code is readily available [Barry et al. 1995].

\section{IMPLEMENTATION}

A Fortran version (both single and double precision) of the above algorithm has been produced [Barry et al. 1995]. The user calls a function called WAPR, supplies the argument $x$, and indicates whether the upper or lower branch is to be used. Alternatively, the user can call WAPR with the argument offset from $-\exp (-1)$. This is recommended for $x$ close to $-\exp (-1)$ to avoid rounding error in the specification of $x$. Also provided is a test program that compares the approximations with "exact" values of the $W$-function, the latter values found using the bisection method applied to (1). Near $x=0$, the "exact" values of $W_{p}$ are calculated using a truncated version of (2). Finally, because the bisection method (or any other root-finding scheme) will be prone to roundoff errors in some circumstances (i.e., near $x=0$ and $x=-\exp (-1)$ ), a list of "exact" $W(x)$ results, correct to 40 significant figures, has been included for independent verification of WAPR. WAPR is written in Fortran and has been run successfully on popular platforms (e.g., Sun Sparc II, 486PC, DEC Alpha, Cray X/MP).

\section{ACKNOWLEDGMENT}

D. A. Barry acknowledges the support of the Australian Research Council.

\section{REFERENCES}

Barry, D. A., Barry, S. J., AND Culligan-Hensley, P. J. 1995. Algorithm 743: WAPR: A Fortran routine for calculating real values of the W-function. ACM Trans. Math. Softw. 21, 2. This issue.

Barry, D. A., Parlange, J.-Y., Sander, G. C., and Sivapalan, M. 1993. A class of exact solutions for Richards' equation. J. Hydrol. 142, 1-4 (Feb.), 29-46.

Char, B. W., Geddes, K. O., Gonner, G. H., Leong, B. L., Monagan, M. B., and Watt, S. M. 1991. Maple V Library Reference Manual. Springer-Verlag, New York.

De BRuiJn, N. G. 1958. Asymptotzc Methods in Analysts. North-Holland, Amsterdam, 25-28.

Euler, L. 1921. De Serie Lambertina Plurimisque Eius Ingignibus Proprietatibus. In Leonhardı Eulerı Opera omnia: sub auspiciis Societatıs Sclentıarum Naturalium Helvetıcae, edenda curaverunt F. Rudio, A. Krazer, P. Stackel. Series Prima VI, pp. 350 ff.

Fritsch, F. N., Shafer, R. E., ANd Crowley, W. P. 1973. Solution of the transcendental equation $w e^{w}=x$. Commun. ACM 16, 2 (Feb.), 123-124

GREEN, D. H. AND KNUTH, D. E. 1981. Mathematıcs for the Analysis of Algorithms. Birkhäuser, Boston, Mass., 47-48. 
LoRentzen, L. AND WAADELAND, H. 1992. Continued Fractions with Applications. NorthHolland, Amsterdam, 21-22.

OuIver, F. W. J. 1990. Asymptotic methods. In Handbook of Applied Mathematics, 2nd ed., C. E. Pearson, Ed. Van Nostrand Reinhold, New York, 633.

Pólya, G. And Szegö, G. 1972. Problems and Theorems in Analysis. Vol. 1. Springer-Verlag, Berlin, 146.

Received September 1992; revised May and December 1993, and January 1994; accepted February 1994 